\title{
A case of recurrent pancreatitis complicated by liver abscesses
}

\author{
Shweta Kumar', Febin John" ${ }^{1 *}$, Balaji Yegneswaran ${ }^{2}$ \\ ${ }^{1}$ Dept of Medicine, resident physician, Saint Peters University Hospital, Rutgers-RWJ Medical School, New Jersey, USA. \\ ${ }^{2}$ Dept of medicine, Associate program director, Saint Peters University Hospital, Rutgers-RWJ Medical school, New Jersey, USA.
}

Received: May 2, 2016; Accepted: June 3, 2016; Published: July 14, 2016

*Corresponding author: Febin John, Saint Peters University Hospital, 254 Easton Avenue, New Brunswick, New Jersey 08901, USA; Tel-90853-37-543; Email-johnfebin35@gmail.com

\begin{abstract}
A 32-year-old obese Caucasian woman presented with acute pancreatitis. Her past medical history includes recurrent pancreatitis, pancreatic divisum, laparoscopic gastric banding and alcohol abuse. The hospital course was complicated by multi-focal hepatic abscesses requiring percutaneous drainage. She eventually underwent pancreatic necrosectomy with J tube placement and was discharged to rehabilitative services. This is a rare case of pancreatitis with concomitant liver abscess from Streptococcus anguinosus.
\end{abstract}

Keywords: Pancreatitis; Streptococcus anguinosus; Hepatic abscess

\section{Introduction}

Pyogenic liver abscess (PLA) was described in the 1800's by Hippocrates. Surgical drainage as a treatment modality was developed in the early $20^{\text {th }}$ century by Oschner and Debakey. However, the mortality rate began to decrease only after the introduction of percutaneous drainage in 1980's [1]. Risk factors for PLA include underlying hepatobiliary or pancreatic disease, liver transplant and diabetes $[2,3]$. Some of these risk factors are related to acute pancreatitis, a public health problem with severe morbidity and mortality [4]. We present a patient with acute pancreatitis and PLA.

\section{Case Report}

A 32-year-old obese Caucasian woman presented to our institution with 5-day history of epigastric pain, fever with malaise and chills. She denied nausea, vomiting or heartburn. There was no jaundice, abdominal distension, overt gastrointestinal bleeding or alteration in bowel habits. Her past medical history was significant for recurrent pancreatitis and pancreas divisum. Review of records revealed heavy alcohol intake and smoking for the past 15 years. She underwent laparoscopic gastric banding, cholecystectomy and a pancreatic stent placement in December 2014. The stent was removed 4 months later and soon thereafter she developed the symptoms which brought her to the hospital. On physical examination, she appeared sick with fever of 101 degree Fahrenheit. Except for tenderness in the epigastrium and right upper quadrant, there was no organomegaly or ascites and Murphy's sign was negative.
Laboratory tests revealed hemoglobin of 8.8 (14-17 gm/dL), hematocrit of $26.5 \%$ (42-52\%), white blood cells 17.6 (4.0-11.0 cumm), raised serum amylase 203 (30-131 U/L), lipase 203 (11$82 \mathrm{U} / \mathrm{L}$ ), CRP 290 (0-5 mg/L) and normal liver enzymes. Blood cultures grew streptococcus anginosus. Computed Tomography (CT) of the abdomen suggested acute pancreatitis superimposed on chronic pancreatitis along with pseudocyst formation.

Aggressive intravenous hydration, antibiotics and adequate pain control was initiated. However, over a period of two weeks, she developed hypotension and oliguric renal failure. She was intubated and started on pressors and a CT-abdomen repeated at this time showed multifocal hepatic abscesses, pancreatic necrosis, ascites and diffuse anasarca (Fig 1). Ultrasound guided drainage of hepatic abscesses was performed and a hepatic drain was left in-situ. Along with continuing intravenous fluids and pain medications, broad-spectrum antibiotics (PiperacillinTazobactam and Vancomycin) were started. She improved clinically; was no longer febrile, abdominal pain resolved and was able to come off the pressors and mechanical ventilation. To evaluate the extent of pancreatic inflammation and hepatic abscesses, a third CT was repeated which showed decrease in pancreatic/peripancreatic fluid collections, intra-abdominal fluid and resolution of hepatic abscesses. An explorative laparotomy was conducted with debridement of necrotic pancreatic tissue, drainage of small collections in the retroperitoneum, removal of the hepatic drain and placement of J-tube for facilitation of
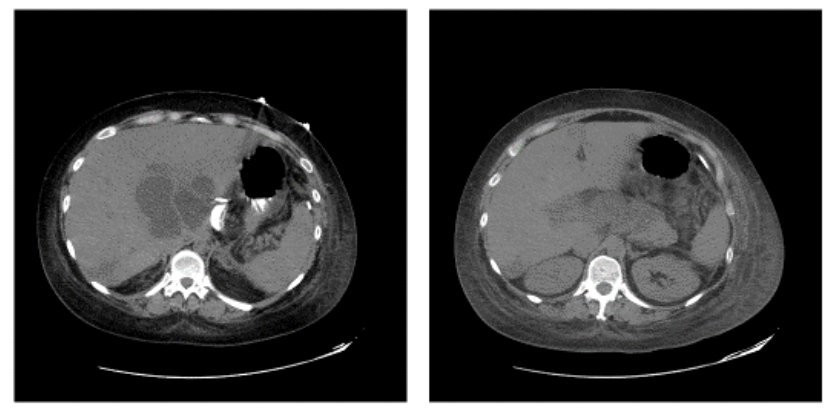

Figure 1: CT image of multifocal hepaticabscess with necrotic pancreas. 
enteral feeding. She was discharged to rehabilitative services on oral pain medications and pancreatic enzyme replacement therapy.

\section{Discussion}

Pyogenic Liver abscess is a potentially lethal problem which can complicate intra-abdominal sepsis of any etiology. PLA occur subsequent to biliary or other intra-abdominal infection or from hematogenous seeding in a systemic infection. Its outcome has improved with modern diagnostic and therapeutic modalities. However, it carries an appreciable mortality rate of about 2-12 percent in developed countries $[1,5]$. The incidence varies between different regions but it is lower in the western world. The mean age is about 55 years with right sided abscess seen in up-to $70 \%$ of patients with PLA. Polymicrobial etiology of PLA have trended towards monomicrobial infection with Klebsiella pneumonia. Streptococcus anguinosus, which is gram positive cocci often, present as normal flora in oral cavity and abdomen is an important etiological agent in the pathogenesis of PLA. It is a highly virulent species notorious for metastatic abscess formation. Its presence should prompt further evaluation for the presence of abscess in different sites.

PLA in the setting of pancreatitis was described as early as 1930's. Pancreatic fistula was described as the mechanism of PLA following an episode of pancreatitis. There was no evidence of pancreato-hepatic fistula in our patient. Recent studies have shown a 3.6-fold increased risk of pancreatitis in patient with PLA, especially in the first 3 months' post infection. This is thought to be from hematogenous spread of undetermined mediators linked to hepatic inflammation. This substantial risk of extensive pancreatic inflammation in patients with PLA is further enhanced by co-morbidities, like hypertriglyceridemia, hepatitis C, biliary stones and alcoholism [6].

There are very few reports of PLA in patients with pancreatitis. The prevalence of streptococcus anguinosus causing PLA in this setting has never been reported. PLA developed during the course of pancreatitis further extended the length of stay in our patient. We conclude that formation of PLA in a patient with pancreatitis can complicate the clinical outcome and hence requires prompt diagnosis and treatment. Further prospective studies should be done to assess this association.

\section{References}

1. Rahimian J, Wilson T, Oram V, Holzman RS. Pyogenic liver abscess: recent trends in etiology and mortality. Clin Infect Dis. 2004;39(11):1654-1659.

2. Huang CJ, Pitt HA, Lipsett PA, Osterman FA Jr, Lillemoe KD, Cameron JL, et al. Pyogenic hepatic abscess. Changing trends over 42 years. Ann Surg. 1996;223(5):600-609.

3. Chan KS, Chen CM, Cheng KC, Hou CC, Lin HJ, Yu WL. Pyogenic liver abscess: a retrospective analysis of 107 patients during a 3-year period. Jpn J Infect Dis. 2005;58(6):366-368.

4. Yadav D, Lowenfels AB. Trends in the epidemiology of the first attack of acute pancreatitis: a systematic review. Pancreas. 2006;33(4):323330.

5. Mohsen AH, Green ST, Read RC, McKendrick MW. Liver abscess in adults: ten years' experience in a UK centre. QJM. 2002;95(12):797802 .

6. Lai SW, Liao KF, Lin CL, Chen PC. Pyogenic liver abscess correlates with increased risk of acute pancreatitis: a population-based cohort study. J Epidemiol. 2015;25(3):246-253. Doi: 10.2188/JE20140152. 